\title{
Immunodeficiency and Microbial Infections
}

\author{
Singh S and Sharma $B^{*}$
}

Department of Biochemistry, University of Allahabad, Allahabad-211002, UP, India

${ }^{*}$ Corresponding author: Sharma B, Department of Biochemistry, University of Allahabad, Allahabad-211002, UP, India, Tel: +91-9415715639, E-mail: sharmabi@yahoo.com

Citation: Singh S, Sharma B (2015) Immunodeficiency and Microbial Infections. J Micro Creat 1(1): 102

Received Date: March 11, 2015 Accepted Date: May 11, 2015 Published Date: May 13, 2015

\begin{abstract}
Immunodeficiency refers to failure of immune system to encounter infections by different microbial pathogens such as fungi, bacteria, viruses and protozoan. This is called acquired or secondary immunodeficiency syndrome (SIS). SID also arises due to other factors such as malnutrition, fatigue, use of immunosuppressants, chemotherapy, skin damage, pregnancy, aging, and recurrent infections. On contrary to it, the primary immunodeficiency syndromes (PIS) are genetic or inherited which makes an individual more susceptible any pathogenic infections. PIS may be recessive and is caused due to mutations in X chromosome. Females therefore act as carriers and in males the disease manifests. This review article illustrates different aspects of immunodeficiency diseases with special reference to microbial infections. The mechanisms of infections by viruses are mentioned with relevant citations. The immunodeficiency syndromes and their associations with different pathogenic factors are summarized in couple of schemes.
\end{abstract}

Keywords: Immunodeficiency syndromes; Microorganisms; Fungi; Bacteria; Viruses; Protozoan; Drugs; Mutations

\section{Introduction}

Immunodeficiency occurs when one or more components of the immune system are defective and hence not working properly. Immunodeficiency is a state in which the immune system fails to fight infectious agents. Most cases of immunodeficiency are acquired (secondary) but some people are born with defects in their immune system (primary) [1]. There are several factors responsible for causing immunodeficiency or immunosuppressant such as malnutrition, fatigue, immunosuppressing agents for organ transplant recipients, chemotherapy for cancer, irradiation, genetic predisposition, skin damage, antibiotic treatment leading to disruption of the physiological microbiome, thus allowing some microorganisms to outcompete others and become pathogenic. For example, disruption of intestinal flora may lead to Clostridium difficile infection and disruption of vaginal flora may lead to Candida infection etc. The imbalance in the levels of physiological microbiome may also lead to leukopenia (i.e. neutropenia and lymphocytopenia), medical procedures, pregnancy and ageing and advanced HIV infection including recurrent infections [2].

The commonest cause of immune deficiency worldwide is malnutrition; however, in developed countries, most immunodeficiency diseases are inherited. The acquired immunodeficiency i.e. secondary immunodeficiency may manifest later in life. In the primary and secondary immunodeficiency, the pathogenic infections influence the functions of different components of the immune system such as those of lymphocytes (T-cell and B-cells) differently. The defects in phagocytic cells or complement fixation cascade or cytokines or their receptors or in molecules that mediate effectors responses have also been reported. Thus, both the innate and the adaptive immune systems can carry defects and may result into immunodeficiency. The inherited diseases are linked to the defective gene. The endeavors to generate clear information about these genes involved in regulation of specific functions of immune systems must be made to develop and understand not only the molecular bases of immune processes but also for providing tools for diagnosis, genetic counseling, and gene therapy. For example, defects in antibody, complement, or phagocyte function are indicative of recurrent infection by pyogenic bacteria, whereas the recurrent viral infections are more indicative of a defect in T-cells associated host defense system [3]. The present article illustrates an updated account of various causes responsible for occurrence of primary and secondary immunodeficiency in humans, involvement of different cellular and genetic components of immune system with special reference to microbial (fungal, bacterial, protozoan and viral) infections. The possible treatment for primary immunodeficiency (due to genetic disorders) is also included.

\section{Categorization of Immunodeficiency Syndromes}

Immunodeficiency syndromes are placed mainly into two distinct categories: (1) Primary Immunodeficiency Syndromes and (2) Secondary Immunodeficiency Syndromes. The primary immunodeficiency syndromes are inherited due to single-gene disorder that appears in infancy or in early childhood (less than 20 years of age) with the exception of common variable immunodeficiency which usually occurs in adults [2]. The genetic or inherited immunodeficiency makes an individual highly susceptible to any pathogenic infections which ultimately lead to death. Many such identified diseases are recessive and are caused by mutations in genes on the X chromosome [4]. In females such defects enable them to act as carriers whereas in males it causes manifestation of a disease because in the former case there are two X chromosomes and in the later there is only one. 


\section{Primary Immunodeficiency Syndromes}

Primary Immunodeficiency Syndromes (PIS) are genetically determined and it occurs due to defects in humoral immunity, cellular immunity, combined humoral and cellular immunity, phagocytic cells and complement proteins etc. The frequency of occurrence of primary immunodeficiency syndromes is $10^{-4}$ [5]. Due to primary immunodeficiency, children suffer from hypogammaglobulinaemia (low numbers of antibodies secreting B cells) [1,6,7] or IgG subclass deficiency or partial antibody deficiency with impaired polysaccharide responsiveness (IPR) or selective IgA deficiency (IgAD) [6]. In the children, the function of the T lymphocytes is also altered due to the genetic defects in the cells. Some workers $[1,8,9]$ have shown that primary genetic immune disorders are due to defects in single gene. Because of defects in T-cells mediated cellular immunity, the individuals in such an immune compromised state can suffer from opportunistic infections by several pathogens including bacteria (Pneumocystis jirovecii), fungi and viruses. In any individual, a rare defect in primary natural killer cells may invite viral infections. Since the functions of T and B-lymphocytes are interdependent, the defects in T-cells result into immunoglobulins deficiencies which may later culminate into an abnormal physiological state with severely combined immunodeficiency (SCID). SCID primarily includes defects in both the B- and T-cells.

\section{PIS due to defect in defense cells}

The defects in the phagocytic cells such as monocytes, macrophages, granulocytes including neutrophils and eosinophils may invite other opportunistic bacterial infections including cutaneous staphylococcal and gram-negative infections. Mycobacteria and salmonellae enter dendrite cells and macrophages where they reproduce and multiply. The entry of these bacterial species is mediated via docking of the bacterial surface lipoproteins with the receptors including Toll-like receptors (especially TLR-2) located on to the surface of these cells. In normal condition, this docking process initiates production of nitric oxide (NO), a toxic substance to bacteria, within these cells. TLRs are known to stimulate release of IL-12 which, in turn, stimulates CD4 T cells to release IFN- $\gamma$ (involved in killing of bacteria) and TNF- $\alpha$. The main function of these cytokines is to activate and recruit more mononuclear phagocytic cells to the site of infection, causing the formation of granulomas [10]. Any defect in the receptors may adversely influence the pathogen neutralization processes by these cells which may enhance the susceptibility of the individuals to pathogenic infections, particularly by mycobacteria and salmonellae. It arises due to the null mutations in one of the following genes: IL-12, the IL-12 receptor $\beta 1$ chain, or either of the two protein subunits, R1 and R2, of the receptor for IFN- $\gamma$ [11,12]. Further, the defects in complement fixation of immune system in any individual can adversely impact its classical and/or alternate pathways resulting into defective humoral immune function. Primary immunodeficiency may also occur due to defects in T-cell signaling, cytokines production and their actions $[13,14]$.

\section{PIS due to defect in defense cells induced by viral infections}

Infection with Epstein-Barr virus (EBV), a herpes virus infecting the humans can transform B lymphocytes and may induce immunodeficiency. After primary infection, EBV stays latent in B lymphocyte cells for longer period. However, T-cell immunodeficiency may develop a potentially lethal B-cell lymphoma. It has been reported that B-cell lymphoma develops due to mutations in 1A (SH2D1A) gene encoding a protein with SH2-domain. This domain interacts with the cytoplasmic tails of two transmembrane receptors such as signaling lymphocyte activation molecule (SLAM) and 2B4 and to the T-cell adhesion molecule, CD2. SLAM is expressed on activated T cells, whereas 2B4 is found on T cells, B cells, and NK cells. Activation of these receptors initiates a signaling pathway by the recruitment of the tyrosine phosphatase, SHP-2. It appears that the function of SH2D1A is to inhibit the recruitment of SHP-2 and thereby to inhibit cellular activation by SLAM and 2B4. SH2D1A is implicated in tumor suppression in addition to restricting a virus to cause the tumor formation [15,16]. Primary SLAM is expressed on activated T cells, whereas 2B4 is found on T cells, B cells, and NK cells. Activation of these receptors initiates a signaling pathway by the recruitment of the tyrosine phosphatase, SHP-2. It appears that the function of SH2D1A is to inhibit the recruitment of SHP-2 and thereby to inhibit cellular activation by SLAM and 2B4. SH2D1A is implicated in tumor suppression in addition to restricting a virus to cause the tumor formation $[15,16]$.

\section{Common Symptoms of SIS}

Some of the apparent warning signals, which arise due to primary immunodeficiency may include (i) eight or more new ear infections or two or more sinus infections or two or more pneumonias within one year, (ii) long term (two or more months) usage of antibiotics with little or no effects or need for intravenous antibiotics to eradicate microbial infections, (iii) failure in weight gain or growth by an infant, (iv) frequent deep skin or organ abscesses or numerous deep-seated infections, (v) persistent thrust in mouth or elsewhere on skin after lyear of age and (vi) a family history of primary immunodeficiency.

\section{Secondary Immunodeficiency Syndromes}

The Secondary Immunodeficiency Syndromes (SIS) occur in those patients suffering from lymphoreticular malignancy, malnutrition, metabolic disorders (renal disease), trauma or major surgery, protein loss - for example, due to nephritic syndrome, systemic disorders (eg, diabetes, undernutrition, HIV infection), treatment with immunosuppressive agents (eg, cytotoxic chemo- 
therapy, bone marrow ablation before transplantation, radiation therapy), treatment with immunomodulatory agents such as rituximab (affecting B-cells) and infliximab, etanercept, adalimumab, anakinra (affecting cellular immunity), drug-induced hypogammaglobulinemia (caused by certain antiepileptics such as diphenylhydantoin, carbamazepine, valproate etc.), metabolic diseases such as diabetes, hepatic disorders and uremia as well as the prolonged serious illness etc. Secondary immunodeficiency may also take place in certain clinical conditions such as prolonged serious illness which may impair immune responses; loss of serum proteins (particularly IgG and albumin) through the kidneys in nephritic syndrome, through skin in severe burns or dermatitis, or through the GI tract in enteropathy. Other factors responsible for occurrence of secondary immunodeficiency include defects in endocrine factors causing diabetes mellitus; gastrointestinal infections causing hepatitis; hematologic defects causing sickle cell disease, myeloma and lymphoma; application of drugs (cytotoxic drugs, anticonvulsants, immune-suppressants, steroids, antibiotics, monoclonal antibodies etc.); nutritional disorders such as alcoholism and undernutrition; physiologic disorders causing immaturity of the immune system in infants and due to pregnancy; renal defects causing uremia and nephritic syndrome etc [17-19].

\section{SIS and microbial infections}

In addition, secondary immunodeficiency can be caused due to microbial (fungal, bacterial and viral). The infections due to viruses such as cytomegalovirus [20], Epstein-Barr virus [21], HIV-1 [22-24], measles virus [25], varicella-zoster virus [26], Polyomavirus, Kaposis Sarcoma virus [27], and Human herpesvirus [28] bacterial super antigens (antigens that can activate large numbers of T cells, resulting in massive cytokine production, most notably from Staphylococcus aureus, Staphylococcus aeruginosa, Staphylococcus pneumonia, Staphylococcus pyogenes, Mycobacterium avium complex (MAC), Pneumocystis jirovecii, Toxoplasma gondii etc.; fungi such as Acinetobacter baumanni, Aspergillus niger sp., Candida albicans, Clostridium difficile, Cryptococcus neoformans, Cryptosporidium, Geomyces destructans, Histoplasma capsulatum, Isospora belli, Legionella pneumophila and Microsporidium are reported to cause secondary immunodeficiency in an individual. The fungal infections causing immunodeficiency include the pathogens such as Candida and Aspergillus species; the mechanisms of action of each of these causal microorganisms may be different and for many of these they are yet to be explored [29]. The different points of origins of primary and secondary immunodeficiency with special reference to pathogenic infections are summarized in Scheme-1. Also, a summary of several common infections associated to immunodeficiency caused by varied microorganisms are displayed in Scheme-2.

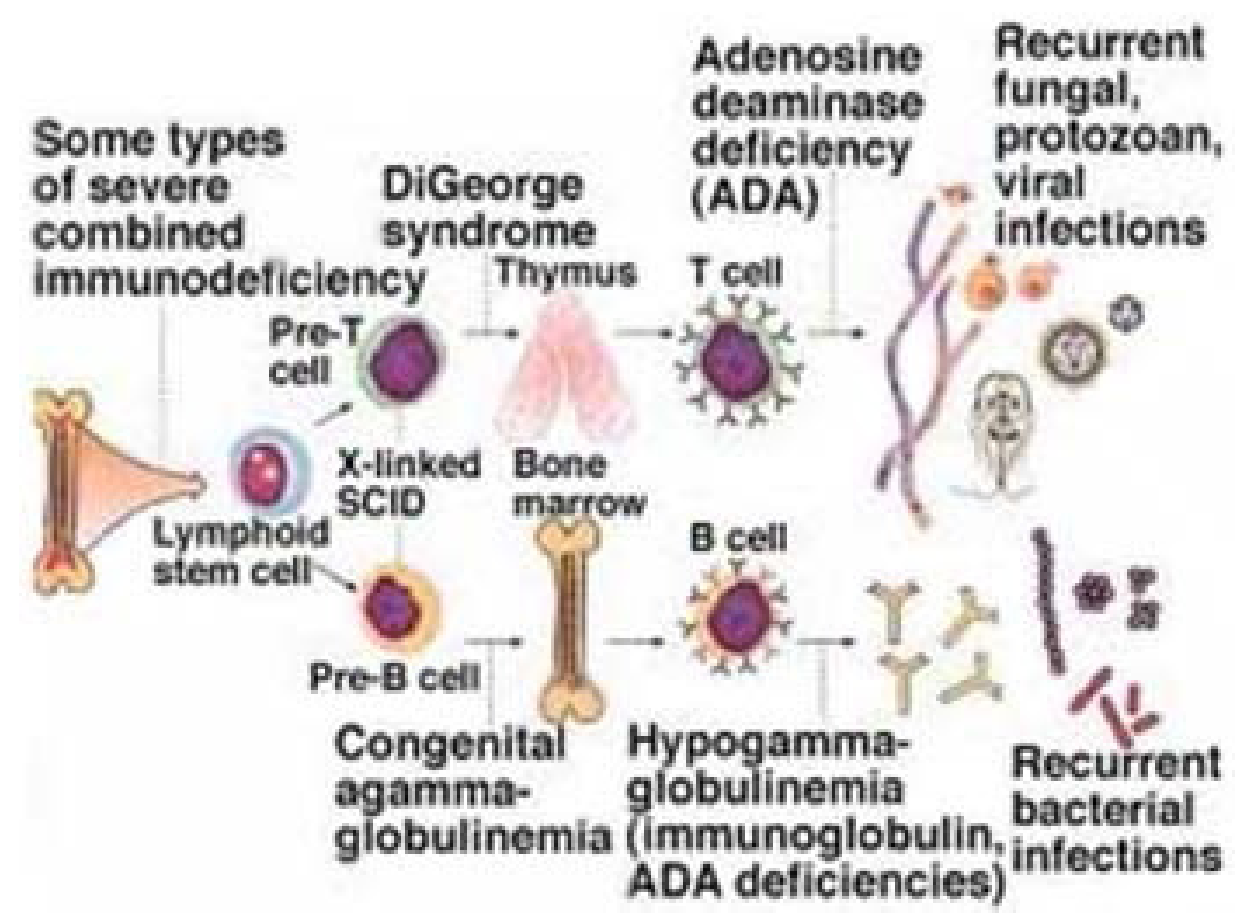

Scheme 1: Points of primary and secondary immunodeficiencies origins and microbial infections 


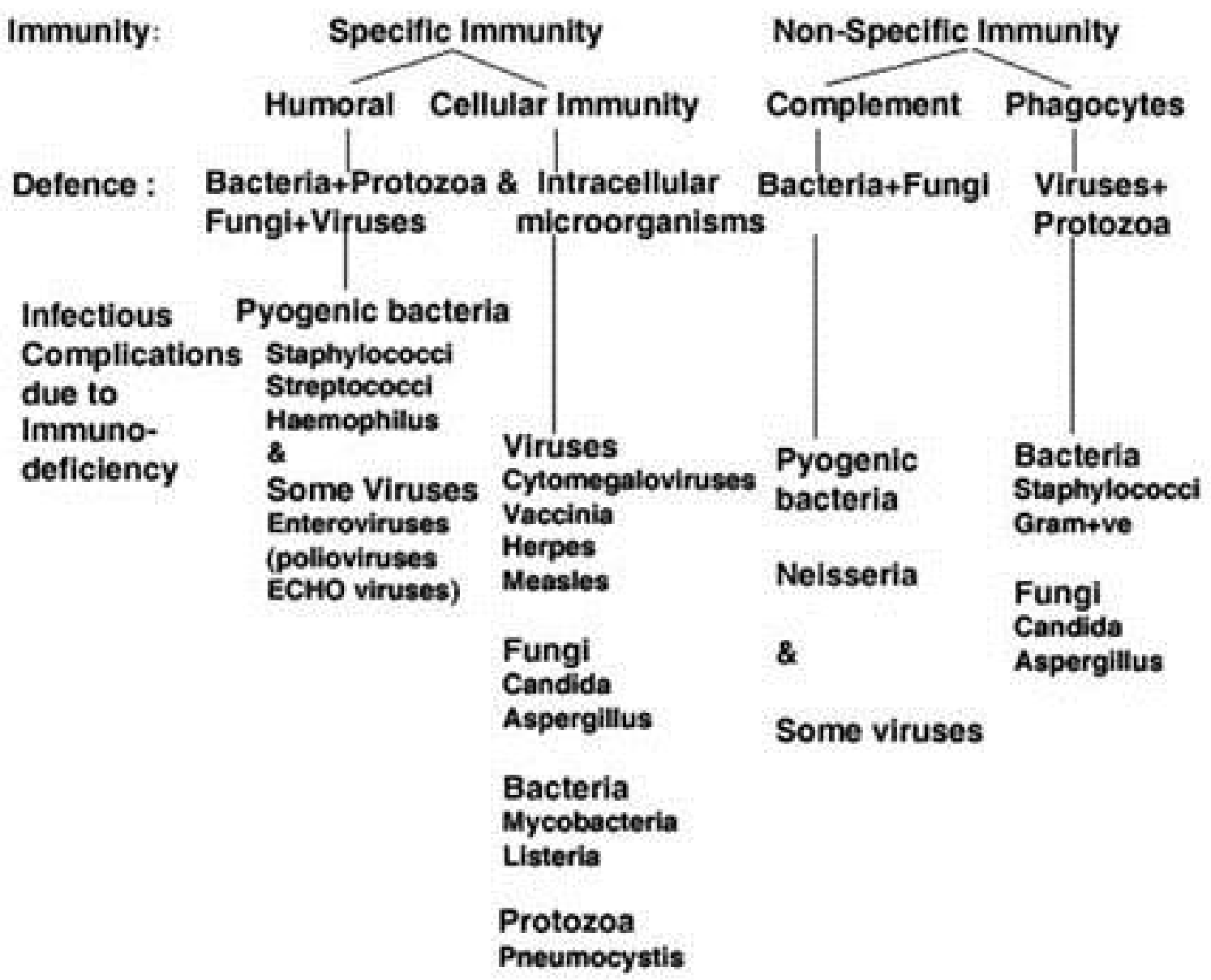

Scheme 2: Common infections associated to immunodeficiency caused by different microorganisms

\section{Acknowledgement}

One of the authors (SS) is grateful to UGC-New Delhi, India for financial support in the form of Dr. D. S. Kothari Post Doctoral Fellowship award.

\section{References}

1. Wood PM (2010) Primary antibody deficiency syndromes. Curr Opin Hematol 17: 356-61.

2. Stray-Pedersen A, Abrahamsen TG, Froland SS (2000) Primary immunodeficiency diseases in Norway. J Clin Immunol 20: $477-85$.

3. Pan-Hammarstrom Q, Hammarstrom L (2008) Antibody deficiency diseases. Eur J Immunol 38: 327-33.

4. Lougaris V, Ferrari S, Cattalini M, Soresina A, Plebani A (2008) Autosomal recessive agammaglobulinemia: novel insights from mutations in Ig-beta. Curr Allergy Asthma Rep 8: 404-8.

5. Dube DS, Chi DS, Hu JY, Krishnaswamy G (2002) An update on their multiple causes, manifestations, and evaluation When to suspect an immunodeficency disorder and how to manage the disease. P G Med 112.

6. Stiehm RE (2007) The four most common pediatric immunodeficiencies. Adv Exp Med Biol 601: 15-26.

7. Moise A, Nedelcu FD, Toader MA, Sora SM, Tica A, et al. (2010) Primary immunodeficiencies of the B lymphocyte. J Med Life 3: 60-3.

8. Kobrynski LJ, Sullivan KE (2007) Velocardiofacial syndrome, DiGeorge syndrome: the chromosome 22q11.2 deletion syndromes. Lancet 370: 1443-52.

9. Routes JM, Grossman WJ, Verbsky J, Laessig RH, Hoffman GL, et al. (2009) Statewide newborn screening for severe T-cell lymphopenia. JAMA 302: 2465-70.

10. Roy-Ghanta P (2010) Immunodeficiency (Primary and Secondary). emis: 1-4.

11. Fischer A (2004) Human primary immunodeficiency diseases: a perspective. Nat Immunol 5: 23-30.

12. Goyal R, Bulua AC, Nikolov NP, Schwartzberg PL, Siegel RM (2009) Rheumatologic and autoimmune manifestations of primary immunodeficiency disorders. Curr Opin Rheumatol 21: 78-84.

13. Roy-Ghanta S, Orange JS (2010) Use of cytokine therapy in primary immunodeficiency. Clin Rev Allergy Immunol 38: $39-53$.

14. Cazander G, Jukema GN, Nibbering (2012) Complement Activation and Inhibition in Wound Healing. Clini Develop Immunol doi: 10.1155/2012/534291.

15. Cai Q, Chen K, Young KH (2015) Epstein-Barr virus-positive T/NK-cell lymphoproliferative disorders. Experi Mole Med 47: 133.

16. Young COK, Ling Li L, Young KH (2015) EBV-driven B-cell lymphoproliferative disorders: from biology, classification and differential diagnosis to clinical management. Experi Mole Med 47: e132. 
17. Santos JI (1994) Nutrition, infection, and immunocompetence. Infect Dis Clin North Am 8: 243-67.

18. Griffin DE (2010) Measles virus-induced suppression of immune responses. Immunol Rev 236: 176-89.

19. Kaymakcalan MD, Je Y, Sonpavde G, Galsky M, Nguyen PL, et al. (2013) Risk of infections in renal cell carcinoma (RCC) and non-RCC patients treated with mammalian target of rapamycin inhibitors. Br J Cancer 108: 2478-84.

20. Schleiss MR (2011) Congenital cytomegalovirus infection: molecular mechanisms mediating viral pathogenesis. Infect Disord Drug Targets 11: $449-65$.

21. Chesnokova LS, Hutt-Fletcher LM (2014) Epstein-Barr virus infection mechanisms. Chin J Cancer 33: 545-8.

22. Douce VL, Herbein G, Rohr O, Schwartz C (2010) Molecular mechanisms of HIV-1 persistence in the monocyte-macrophage lineage. Retrovirology 7: 32.

23. Sharma B (2014) Oxidative stress in HIV patients receiving antiretroviral therapy. Curr HIV Res 12: 13-21.

24. Sharma B (2015) Drug Resistance in HIV-1: Genetic and Molecular Bases, Mechanisms and Strategies to Combat the Issue. Biochem Analyt Biochem 4: e153.

25. Xuelian B, Ghildyal R (2012) Measles Virus infection: mechanism of immune suppression. In Medicine: Immunology, allergology and Rheumatology: Immunosuppression-Role in Health and Diseases. ed Kapur S and Portela MB 12: 225-31.

26. Zerboni L, Sen N, Oliver SL, Arvin AM (2014) Molecular mechanisms of varicella zoster virus pathogenesis. Nat Rev Microbiol 12: 197-210.

27. Chapagain ML, Nerurkar VR (2010) Human polyomavirus JC (JCV) infection of human B lymphocytes: a possible mechanism for JCV transmigration across the blood-brain barrier. J Infect Dis 202: 184-91.

28. Alibek K, Baiken Y, Kakpenova A, Mussabekova A, Zhussupbekova S, et al. (2014) Implication of human herpesviruses in oncogenesis through immune evasion and suppression. Infec Agent Cancer 9: 3.

29. Kamel OW, van de Rijn M,Weiss LM, Del Zoppo GJ, Hench PK, et al. (1993) Brief report: reversible lymphomas associated with Epstein-Barr virus occurring during methotrexate therapy for rheumatoid arthritis and dermatomyositis. N Engl J Med 328: 1317-21.

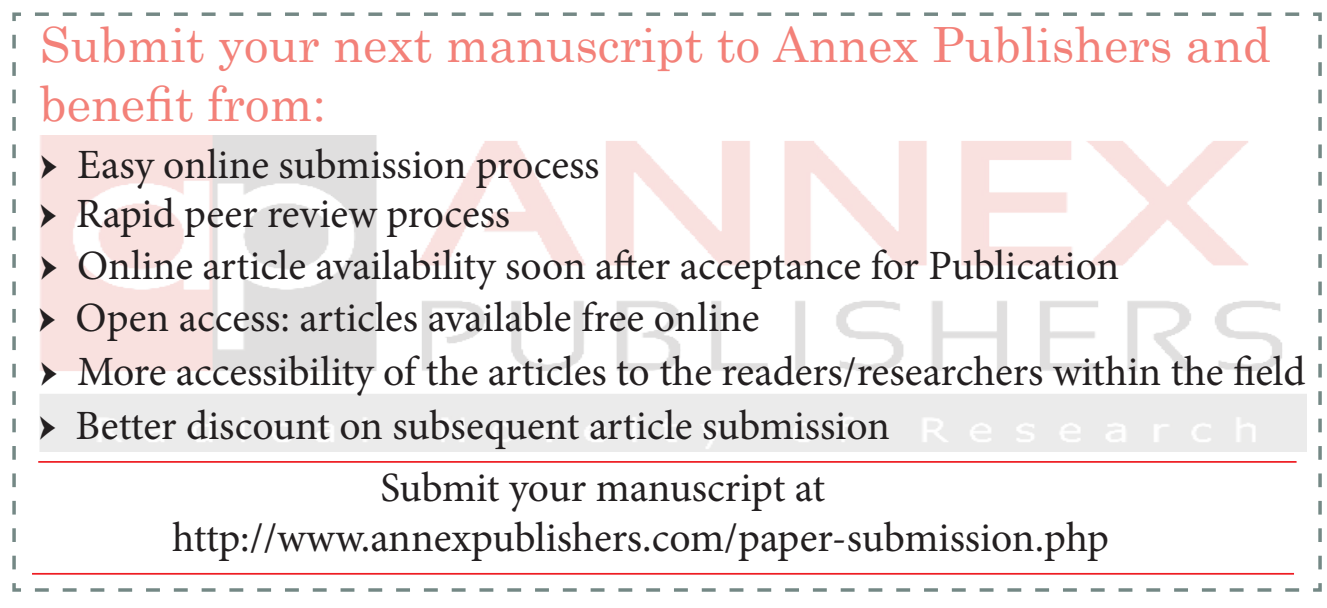

